\title{
Economic benefit of leptospirosis prevention in Kelantan, Malaysia: willingness- to-contribute approach
}

\begin{abstract}
Leptospirosis is an endemic disease in Malaysia. Despite the increasing incidence rate, knowledge on the economic assessment of preventing leptospirosis is still limited. This paper introduces the willingness-to-contribute (WTC) method for estimating the economic benefit of preventing leptospirosis. A cross-sectional study using the WTC method was applied to measure how much time respondents in Kelantan were willing to contribute toward preventing leptospirosis. Study respondents were wet market traders aged 18 years old and above who were fluent in the Malay language. The average WTC value was multiplied by the population of Kelantan to derive the monetary value of preventing leptospirosis. Two hundred and fifty respondents participated in the study. The mean time contribution was 6.68 hours (SD9.01) per month. The average WTC corresponded to a monthly cost savings of US $\$ 4.94$ per person. Approximately between US\$106.7 million to US\$315 million per annum can be saved through the prevention of leptospirosis in Kelantan. Preventing leptospirosis is beneficial to Kelantan and would bring major economic savings. The findings are intended to help policy makers in the planning and management of leptospirosis policies and interventions.
\end{abstract}

Keyword: Malaysia; Contingent valuation method; Economic assessment; Leptospirosis; Willingness-to-contribute 
